\title{
El uso de los errores como herramienta del aprendizaje autorregulado en estudiantes de secundaria
}

\author{
Ángela Zamora Menéndez(*), José Manuel Suárez Riveiro(**) y Diego Ardura(***) \\ $\left.{ }^{*}\right)$ Universidad Nacional de Educación a Distancia; ${ }^{(* *)}$ Universidad Internacional de la Rioja y ${ }^{* * *}$ Colegio Santo Domingo de Guzmán-FESD
}

\begin{abstract}
RESUMEN
Este estudio tuvo como objetivo investigar las relaciones entre la facilidad con que los estudiantes de ciencias de secundaria detectan sus errores, su precisión en la autoevaluación y las variables motivacionales y cognitivas implicadas en el proceso de autorregulación. Tanto en la variable de detección de errores como la de autoevaluación se estudió el efecto de la utilización de guiones de evaluación. En la investigación participaron un total de 151 estudiantes de secundaria. Las variables cognitivas y motivacionales se midieron empleando el cuestionario MSLQ. Los resultados indican que no existe una relación directa entre los procesos de autoevaluación y los de detección de errores. Durante estos últimos se invocan más estrategias cognitivo-motivacionales que en los primeros. El uso de los guiones de auto-evaluación mejora ambos procesos.
\end{abstract}

Palabras Clave: enseñanza secundaria, autoevaluación, motivación, estrategias de aprendizaje, autoaprendizaje.

\section{The use of errors as a tool for self-regulated learning in secondary school students}

\section{ABSTRACT}

The aim of this study was to investigate the relationships between the ease with which students detect their own mistakes. Their selfassessment accuracy and the cognitive and motivational variables involved in the self-regulation cycle. The use of a self-assessment script was studied both for self-assessment and error detection. A total of 151 secondary students took part in the investigation. The cognitive and motivational variables were measured using the MSLQ questionnaire. Correlational, cluster and differential analyses were employed in this study. We found no relationship between the former and the latter. There were more cognitive and motivational strategies invoked during error searching than in the self-assessment estimation of the grade. Besides, the use of the self-assessment script improved both aspects.

Keywords: secondary education, self-assessment, motivation, learning strategies, self-learning.

\section{Introducción}

Aunque tradicionalmente los errores cometidos por los estudiantes se han considerado un obstáculo para el proceso de enseñanza-aprendizaje, desde una perspectiva constructivista se revelan como un potencial impulsor del mismo (Astolfi, 1999). Cuando se acude a un enfoque sancionador para el tratamiento de los errores cometidos por los alumnos, se pierde gran parte de su potencial educativo. Sin embargo, cuando nos alejamos de este enfoque punitivo, el error puede llegar a convertirse en una herramienta más para el aprendizaje (Schank, 1997). Por tanto, el papel de los errores puede ser clave en el aprendizaje.

La evaluación es tan importante en el proceso de enseñanza-aprendizaje que condiciona no solo qué se aprende sino también cómo se aprende. Dependiendo del carácter de la misma, el error puede ejercer funciones muy diferentes. Mientras que, en el caso de la evaluación sumativa, el error presenta una connota- ción negativa, en el de la evaluación formativa puede constituir una oportunidad para aprender y mejorar. La evaluación formativa es fundamental a la hora de abordar la función reguladora.

Tradicionalmente se le ha dado al docente la responsabilidad de regular el aprendizaje del alumnado. Sin embargo, lo verdaderamente deseable, en la idea conseguir que el alumnado ocupe un espacio central en el proceso de enseñanza-aprendizaje es que sea el propio estudiante quien regule su propio aprendizaje, con la asistencia del profesor. Esto es lo que Nunziati (1990) denomina evaluación formadora. En esta vertiente de la evaluación, la capacidad del estudiante para la detección, análisis y gestión de sus propios errores constituye un recurso esencial para el aprendizaje.

A la hora de abordar esta última tarea, el constructo denominado aprendizaje autorregulado juega un papel fundamental. Según el modelo propuesto por Zimmerman (2000), el ciclo del aprendizaje autorregulado se organiza en tres etapas: previa, de 
realización y de autorreflexión. Como es lógico, en este ciclo, la autoevaluación (Alonso y Panadero, 2010; Baas, Castelijns, Vermeulen, Martens y Seger, 2015; Baars, Vink, van Gog, de Bruin, y Paas, 2014; Hattie, 2013; Panadero y Alonso, 2013; Panadero y Jonsson, 2013) y la gestión de los errores por parte de los estudiantes, puede ser de gran importancia ya que, cuando se comete un error, sería deseable que se desencadenase un procedimiento que permitiese al estudiante encontrar las causas de lo que está sucediendo y solventar la situación. Por tanto, el aprendiz se prepararía para detectar sus dificultades y tratar de autorregular su aprendizaje. En caso de no lograrlo, tendría que considerar la posibilidad de buscar ayuda para poder continuar (Briceño, 2009; González y Paolini, 2015; Mathabate y Potgieger, 2014; Schunk y Zimmerman, 2013).

El aprendizaje autorregulado tiene como elementos básicos para encarar una tarea, por un lado, los aspectos afectivo-motivacionales y, por otro, las estrategias de aprendizaje al alcance del estudiante. En primer lugar, la motivación es un aspecto fundamental en el aprendizaje y constituye un aspecto crucial en todos los modelos para el aprendizaje autorregulado (Cueli, García y González-Castro, 2013; Núñez, Solano, González-Pienda y Rosário, 2006; Rosário, 2004; Zimmerman, 2000). Efectivamente, el alumnado con altos niveles de autorregulación se encuentran motivados e implicados a nivel personal. Además, son tenaces para alcanzar los objetivos ante las tareas que se proponen. Por tanto, aquellos estudiantes que saben autorregularse correctamente, perciben el aprendizaje como un proceso controlable y en el que pueden influir de una manera sistemática. Si, además, relacionan sus éxitos o fracasos a través de atribuciones causales que relacionan con procesos estratégicos que consideran mejorables, el estudiante aumentará sus sensaciones de autoeficacia $\mathrm{y}$, posiblemente, generará un mayor interés por la tarea (Núñez et al., 2006). En segundo lugar, los requerimientos básicos del aprendizaje autorregulado obligan a que el estudiante sea consciente de la demanda de la tarea a realizar, de sus propias potencialidades a nivel personal y de las estrategias que debe emplear para realizarla. En este sentido, la eficacia en la adquisición, procesado, recuperación y transferencia de la información son fundamentales a la hora de encarar una tarea (Barca-Lozano, Almeida, Porto-Rioboo, Peralbo-Uzquiano y Brenlla-Blanco, 2012).

En los últimos años, el estudio de cómo los estudiantes utilizan sus errores para aprender ha recibido cierta atención. La investigación llevada a cabo por Black y William (1998) muestra que el hecho de devolver a los estudiantes sus trabajos acompañados de comentarios, sin indicar la puntuación implica una mejoría en el rendimiento de los estudiantes. Por el contrario, cuando los trabajos se devuelven corregidos pero indicando únicamente la nota, no se produce mejoría. En esta misma línea, esta investigación ha encontrado que, cuando se devuelven los trabajos o pruebas indicando la calificación y añadiendo comentarios, no se aprecia una mejora en el aprendizaje de los estudiantes, puesto que focalizan su atención, no en los comentarios del profesor, sino en la nota que les ha otorgado.

En el campo de la enseñanza de la física existen trabajos recientes que ponen de manifiesto el interés que podría tener la gestión de los errores por parte de los estudiantes como una oportunidad de aprendizaje. Por ejemplo, para tratar de utilizar los errores como medio didáctico, Henderson y Harper (2009) proponen el uso de las correcciones que llevan a cabo los estudiantes del grado en física sobre sus pruebas escritas. La meta de este procedimiento es que los estudiantes sean autónomos a la hora de encontrar sus propios fallos y que sean capaces de inferir una generalización que les ayude en su aprendizaje. Por otro lado, los resultados de Mason y Singh (2010) y Yerusalmi, Cohen,
Mason y Singh (2012a, 2012b) señalan que los universitarios de física no utilizan automáticamente sus errores como un recurso para el aprendizaje. Además, ese aprovechamiento de los errores, parece un proceso complejo y que, en general, no tiene lugar espontáneamente pues aquellos estudiantes que reciben asesoramiento, son los que finalmente detectan y describen mejor sus errores.

Estudios recientes (Zamora y Ardura, 2014; Zamora, Suárez y Ardura, 2017, 2018) ha confirmado los hallazgos comentados anteriormente y ha demostrado la efectividad del trabajo con los errores para el rendimiento de estudiantes de ciencias de secundaria. La principal conclusión de estas investigaciones es que aquellos estudiantes que, además de diagnosticar sus errores, llevan a cabo un trabajo posterior de autorregulación retroactiva son los que, en mayor medida, mejoran su rendimiento durante la experiencia. Teniendo en cuenta además que, de acuerdo con esta y otras investigaciones anteriores (Zamora et al., 2014; Zamora et al., 2017, 2018; Yerusalmi, Cohen, Mason y Sigh, 2012a; 2012b), la utilización de los errores por parte de los estudiantes durante su aprendizaje es complejo y no tiene lugar espontáneamente, estos resultados apuntan a que es necesario de un lado, un cambio del estatus del error en el aprendizaje.

\section{Método}

\section{Objetivos}

Dada la importancia de las estrategias de aprendizaje y de la motivación en el aprendizaje autorregulado y el importante papel que pueden desempeñar la autoevaluación y el manejo de los errores en este último, el objetivo de este trabajo es investigar las relaciones existentes entre todas estas variables mediante un análisis correlacional. Además, se comprobará el efecto de las mismas en la detección, por parte de los estudiantes, de sus propios errores empleando una investigación diferencial.

\section{Muestra}

En la investigación participaron un total de 151 estudiantes que cursaban $2^{\underline{0}}$ y $3^{\circ}$ de Educación Secundaria Obligatoria en un centro educativo español. Los estudiantes participaron de forma voluntaria y dieron su consentimiento informado. La experiencia se llevó a cabo en una asignatura de Ciencias Naturales y en el contexto de una unidad didáctica sobre las leyes de los gases. Los estudiantes fueron asignados alfabéticamente a 8 aulas por parte del centro educativo al principio del curso. Para seleccionar los grupos que han participado en la investigación se ha utilizado el criterio de disponibilidad y accesibilidad tanto de los estudiantes como del docente, por lo que se aplicó un muestro por conveniencia. La media de edad fue de 13,65 años (moda $=14$ y mediana $=14)$. Un total de 82 estudiantes $(53,30 \%)$ eran chicos y 69 estudiantes $(45,70 \%)$ eran chicas.

\section{Instrumento}

Las medidas de las variables estratégicas y motivacionales incluidas en esta investigación se llevaron a cabo utilizando el Motivated Strategies Learning Questionnaire (MSLQ) (Pintrich y García, 1991; Pintrich, Smith, García, y McKeachie, 1991). El cuestionario estaba formado por 37 ítems medidos en una escala tipo Likert de 7 puntos. Este cuestionario se divide en dos subescalas. La primera de ellas busca evaluar los aspectos motivacionales y la segunda permite extraer información sobre las estrategias de aprendizaje que utilizan los estudiantes. Después 
de la validación del instrumento, el cuestionario, la subescala de motivación quedó formada por 15 ítems que se organizan en cuatro factores que dan cuenta de: (a) la orientación a metas extrínsecas, (b) el valor de la tarea, (c) las creencias de autoeficiacia y (d) la ansiedad en situaciones de examen. Estos factores explican el 61,53\% de la varianza muestral, alcanzando una fiabilidad, medida mediante el alfa de Cronbach, de 0,62 (véase la Tabla 1). La subescala de estrategias de aprendizaje quedó conformada por 22 ítems que dieron cuenta de un 63,25\% de la varianza y una fiabilidad de 0,80. Esto ítems quedaron agrupados en siete factores que evalúan los siguientes aspectos: (a) estrategia de elaboración, (b) estrategia de organización, (c) pensamiento crítico, (d) autorregulación de la metacognición, (e) gestión del tiempo y lugar de estudio, (f) regulación del esfuerzo y (g) aprendizaje de otros compañeros.

\section{Variables}

Además de las variables motivacionales y cognitivas indicadas anteriormente, en esta investigación se han estudiado dos variables: la detección de errores y el desajuste en la autoevaluación.

En esta investigación, para medir la facilidad con que los estudiantes encuentran sus propios errores, se han definido dos variables. Por un lado, se define la detección de errores sin ayuda (DESA) como la facilidad con la que los estudiantes detectan sus propios errores sin el uso del guion de evaluación después de realizar el examen. Por otro lado, se ha definido una variable denominada detección de errores con ayuda (DECA) como la facilidad con la que los estudiantes encuentran sus propios errores con el apoyo del guion de evaluación propuesto por el docente para evaluar la prueba. Para medir ambas variables se utilizó el mismo procedimiento: los estudiantes utilizaron un registro para anotar los errores detectados que contaba con un espacio para que especificasen, los errores que habían identificado en la prueba. A continuación se calculó el porcentaje de errores que cada estudiante había detectado, tomando como referencia los errores totales detectados por el docente. De esta manera, los porcentajes obtenidos nos permiten medir la progresión del alumno desde que hace su valoración con sus propios recursos hasta cuando tiene su disposición el guion de evaluación.

Para cuantificar la precisión en las autoevaluaciones de los estudiantes, se definieron dos variables, denominadas desajuste en la autoevaluación, en las que se comparó la nota con el que el estudiante con la calificación del docente en los dos casos referidos anteriormente: sin la ayuda del guion de evaluación (DASA) y con la ayuda del mismo (DACA).

\section{Procedimiento de recogida de datos}

Los participantes respondieron el MSLQ con el objetivo de conocer su motivación y las estrategias de aprendizaje que utilizaban. Posteriormente, el docente de la asignatura explicó los aspectos conceptuales del tema. En la siguiente sesión y, una vez concluida la explicación teórica, para evaluar el grado de comprensión de los contenidos, los estudiantes realizaron una prueba de evaluación con preguntas teóricas y prácticas. La prueba fue devuelta a los participantes para que localizaran en ella los errores cometidos. Para no alterar el trabajo de detección de errores de los estudiantes, se les entregó la prueba sin evaluar y sin incluir indicaciones o correcciones por parte del profesor. Tras la localización de los errores, los alumnos se autoevaluaron en cada una de las preguntas de la prueba. Para realizar esta tarea, podían utilizar únicamente el libro de texto y las anotacio- nes registradas durante las clases y, además de contar con los resultados numéricos finales de los ejercicios prácticos pero, en ningún caso, podían consultar al profesor ni a los compañeros. Posteriormente, se les suministró a los estudiantes un guion de evaluación, en el que se indicaban las respuestas para que una pregunta se considerase correcta, para que, nuevamente, volviesen a autoevaluar su trabajo y detectaran sus errores.

\section{Análisis de datos}

En las pruebas de normalidad, únicamente las dos variables de detección de errores (DESA y DECA) exhibieron un comportamiento normal. Por ello, en el caso de los análisis que afectaron a las demás variables se utilizaron las pruebas propias de la estadística no paramétrica. Para los análisis correlacionales se utilizaron, por tanto, los coeficientes de correlación de Pearson para aquellas parejas que implicaban a las primeras y el de Spearman para las que implicaban a alguna de las segundas. Estos coeficientes varían entre 0 y 1 y en el contexto de la investigación educativa se consideran relevantes aquellos que están por encima de 0,4 . Para realizar los análisis de diferencias de medias, se agrupó a los sujetos de la muestra mediante un análisis de conglomerados de k-medias, según los valores de sus estrategias de aprendizaje y su motivación. Alternativamente se agrupó a los estudiantes en función de las variables de desajuste en la autoevaluación (DASA y DACA) en tres grupos homogéneos en función de los niveles de los estudiantes en estas variables. Las diferencias de medias se llevaron a cabo mediante el análisis de la varianza que empleó el estadístico H de Kruskall Wallis. En este caso, se realizaron los análisis post hoc empleando la prueba no paramétrica U de Mann-Whitney aplicando, además, la corrección de Bonferroni (Field, 2009). Los análisis de datos se realizaron mediante el programa SPSS (Statistical Package for Social Sciences) versión 20.0.

\section{Resultados}

\section{Análisis descriptivos y correlacionales}

En la Tabla 1 se presentan los índices de fiabilidad de las escalas estratégico-motivacionales, las medias, las desviaciones típicas y las correlaciones entre variables. Como se puede observar la variable motivacional con un mayor valor medio es el valor de la tarea $(M=5,61)$. Por otro lado, la regulación del esfuerzo $(\mathrm{M}=$ $5,30)$ se erige como la variable de estrategias de aprendizaje con un mayor valor medio. Es interesante advertir que los estudiantes han mostrado unos niveles bajos en las variables de detección de errores tanto sin la ayuda del guion $(\mathrm{M}=18,67)$ como cuando han tenido la posibilidad de utilizarlo $(M=41,16)$.

En este estudio, los estudiantes tuvieron la ocasión de valorar su trabajo en dos momentos consecutivos, pero en los que dispusieron de niveles de información diferentes: en primer lugar, cuando no dispusieron de más ayuda que su propia retroalimentación y sus materiales de aula, se detectó un desajuste medio en su estimación de 1,38 puntos sobre 10. Sin embargo, cuando dispusieron de la posibilidad de utilizar el guion de evaluación este desajuste descendió hasta 0,49 puntos. En ambos casos los estudiantes sobreestimaron su calificación en la prueba (Tabla 1), también se muestran los tantos por ciento de estudiantes que se autoevalúan por exceso, que aciertan su calificación y que se autoevalúan por defecto. Como se puede apreciar, el tanto por ciento de sujetos que sobreestiman su nota decrece cuando utilizan el guion, duplicándose los que aciertan su nota en pos de los que aciertan, pero también se detecta un incremento en el porcentaje de los que la subestiman. 
Tabla 1. Correlaciones entre las variables de la investigación.

\begin{tabular}{|c|c|c|c|c|c|c|c|c|c|c|c|c|}
\hline & $\alpha$ & M & DT & (1) & (2) & (3) & (4) & (5) & (6) & (7) & (8) & (9) \\
\hline OME (1) & 0,60 & 3,26 & 1,66 & 1 & $-0,00$ & $0,40^{* *}$ & $-0,05$ & $-0,07$ & $-0,11$ & 0,01 & $-0,06$ & $-0,00$ \\
\hline VT (2) & 0,77 & 5,61 & 1,05 & & 1 & $-0,12$ & $0,42^{* *}$ & $0,29^{* *}$ & $0,51^{* *}$ & $0,43^{* *}$ & $0,33^{* *}$ & $0,46^{* *}$ \\
\hline A (3) & 0,50 & 4,38 & 1,42 & & & 1 & $-0,39^{* *}$ & $-0,04$ & $-0,21^{* *}$ & $-0,10$ & $-0,21^{* *}$ & $-0,34^{* *}$ \\
\hline AAR (4) & 0,84 & 4,94 & 1,22 & & & & 1 & $0,29^{* *}$ & $0,38^{* *}$ & $0,43^{* *}$ & $0,35^{* *}$ & $0,40^{* *}$ \\
\hline $\mathrm{O}(5)$ & 0,67 & 4,79 & 1,52 & & & & & 1 & $0,47^{* *}$ & $0,37^{* *}$ & $0,29^{* * *}$ & $0,18^{*}$ \\
\hline E (6) & 0,72 & 4,63 & 1,24 & & & & & & 1 & $0,56^{* *}$ & $0,43^{* *}$ & $0,38^{* *}$ \\
\hline PC (7) & 0,73 & 4,13 & 1,37 & & & & & & & 1 & $0,42^{* *}$ & $0,22^{* *}$ \\
\hline AOC (8) & 0,59 & 4,01 & 1,32 & & & & & & & & 1 & $0,24^{* *}$ \\
\hline RE (9) & 0,75 & 5,30 & 1,31 & & & & & & & & & 1 \\
\hline & $\alpha$ & $\mathrm{M}$ & DT & (10) & (11) & (12) & (13) & $(14)^{1}$ & \multicolumn{2}{|c|}{$(15)^{1}$} & & \\
\hline $\mathrm{AM}(10)$ & 0,60 & 4,50 & 1,34 & 1 & $-0,08$ & $-0,03$ & 0,06 & 0,14 & \multicolumn{2}{|c|}{0,08} & & \\
\hline TLE (11) & 0,63 & 3,34 & 1,53 & & 1 & $-0,09$ & $-0,00$ & $-0,12$ & \multicolumn{2}{|c|}{$-0,04$} & & \\
\hline DACA (12) & - & 0,49 & 1,14 & & & 1 & $0,53^{* *}$ & $-0,11$ & \multicolumn{2}{|c|}{$-0,11$} & & \\
\hline DASA (13) & - & 1,38 & 1,60 & & & & 1 & $-0,07$ & \multicolumn{2}{|c|}{$-0,18$} & & \\
\hline $\operatorname{DECA}^{1}(14)$ & - & 41,16 & 30,28 & & & & & 1 & \multicolumn{2}{|c|}{$0,63^{* *}$} & & \\
\hline $\operatorname{DESA}^{1}(15)$ & - & 18,67 & 21,49 & & & & & & \multicolumn{2}{|c|}{1} & & \\
\hline
\end{tabular}

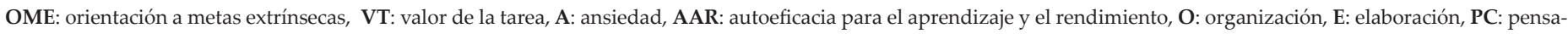

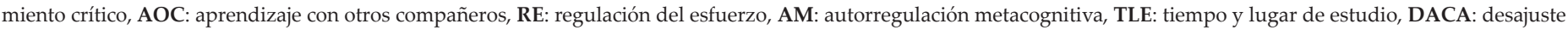

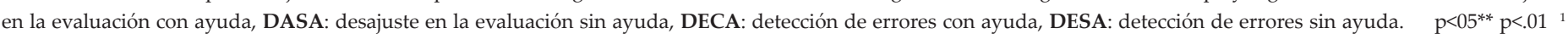
Índice de correlación de Spearman

En relación a los resultados del análisis correlacional, en primer lugar, se describen las relaciones, por un lado, entre las variables motivacionales y estratégicas y, por otro, entre las variables autoevaluación y detección de errores. Finalmente, se presentan las correlaciones entre estos dos grupos de variables. Los análisis de las variables motivacionales implicadas en esta investigación muestran correlaciones significativas. La correlación entre el valor de la tarea y la autoeficacia por un lado, y la motivación extrínseca y la ansiedad por otro, correlacionan positivamente. Sin embargo, en el caso de esta última y la autoeficacia la correlación es negativa. En lo que se refiere a las variables estratégicas, todas las investigadas en este trabajo tienen una relación significativa entre ellas $\mathrm{y}$, a su vez, y con la variable de estrategias metacognitivas -autorregulación-. Esta última se relaciona significativa y positivamente con las estrategias de control de recursos -aprendizaje con otros compañeros y regulación del esfuerzo-. A su vez, estas dos variables estratégicas correlacionan positivamente entre sí y con la estrategia de organización. Por último, la regulación del esfuerzo presenta una correlación negativa con el tiempo y lugar de estudio.

Por otro lado, los resultados confirman la relación existente entre las motivaciones y las estrategias de aprendizaje, ya que todas las variables motivacionales, excepto la orientación motivacional extrínseca, correlaciona bien positiva o negativamente con casi todas las variables referentes a las estrategias de aprendizaje.

Las dos versiones del desajuste en la autoevaluación presentan una correlación significativa y positiva. Por su parte, las variables de detección de errores muestran un comportamiento similar entre ellas (Tabla 1). Los análisis correlacionales indican que también existe una correlación significativa y positiva entre las variables motivacionales y cognitivas y las de detección de errores y las de autoevaluación. En la Tabla 1, como se ha podi- do apreciar, la variable DECA correlaciona significativa y positivamente con la regulación del esfuerzo, el valor de la tarea, la autoeficacia para el aprendizaje y las estrategias de elaboración. De estas, solo la autorregulación del esfuerzo y la autoeficacia correlacionan positivamente con la detección de errores cuando se lleva a cabo sin ayuda (DESA). Únicamente el aprendizaje entre iguales correlaciona positivamente con la autoevaluación con ayuda. Por último, es interesante destacar que la autoevaluación sin ayuda no correlaciona significativamente con ninguna de las variables seleccionadas en este estudio.

\section{La detección de errores: análisis diferencial}

Las variables DECA y DESA se utilizaron en los análisis diferenciales como variables de escala. Todas las demás variables incluidas en este estudio se utilizaron como categóricas. Con las variables cognitivo-motivacionales se construyeron tres grupos empleando un análisis de clúster de k-medias. Para las restantes variables se construyeron tres grupos con aproximadamente el mismo número de sujetos empleando los percentiles correspondientes. Los tres grupos obtenidos mediante el análisis de conglomerados presentan las siguientes características: en un primer grupo que contiene el $35,80 \%$ de los individuos de la muestra, los sujetos presentan un alto grado de autoeficacia y le confieren un valor alto a la tarea; también presentan niveles bajos de motivación extrínseca y de ansiedad. Se trataría, por tanto, de un grupo de estudiantes con alta motivación hacia el aprendizaje. En segundo lugar, se detectó un grupo de estudiantes que constituye el 33,70\% del total de la muestra y que tienen bajos valores en el valor de la tarea, de motivación hacia metas extrínsecas y de autoeficacia acompañados de altos niveles de ansiedad. El tercer y último agrupamiento de contienen un 30,50\% de estudiantes que muestra una ansiedad alta y una elevada motivación extrínseca. 
Además, tienen una autoeficacia alta y confieren un alto valor a la tarea.

El análisis de clúster de las estrategias de aprendizaje permitió agrupar a los estudiantes en tres categorías. Por una parte, el grupo de sujetos de la muestra que presentaban un uso bajo de estrategias de aprendizaje constituyó un 27,20\% de la muestra. Con un nivel medio en cuanto a las estrategias a su alcance se situó un segundo grupo de estudiantes que abarca el 29,10\% de la muestra. Finalmente, un tercer grupo de estudiantes $(43,70 \%)$ que presentó niveles altos en todas las estrategias de aprendizaje incluidas en esta investigación a excepción de la que mide el tiempo y lugar de estudio. En cuanto a las variables de desajuste en la autoevaluación, la muestra se agrupa en tres categorías: bajo, medio y alto, con porcentajes de sujetos de $35,10 \%, 33,80 \%$ y $31,10 \%$ en el caso en el que los alumnos no recibieron ayuda y de $45,60 \%, 27,20 \%$ y $27,20 \%$ cuando los estudiantes reciben la ayuda del guion que suministró el docente.

Tabla 2. Análisis diferencial: prueba de H-test y U-test para muestras independientes.

\begin{tabular}{|c|c|c|c|c|c|}
\hline \multicolumn{6}{|l|}{ Detección Errores Sin Ayuda } \\
\hline Variable de categorización & $\chi^{2}(p)$ & Categorías & Media & Diferencia & $p$ \\
\hline \multirow{3}{*}{ Motivación } & \multirow{3}{*}{$4,27(0,118)$} & MAA & $20,32 \%$ & \multirow{3}{*}{-} & \multirow{3}{*}{-} \\
\hline & & MAR & $23,08 \%$ & & \\
\hline & & $\mathrm{DM}$ & $12,75 \%$ & & \\
\hline \multirow{3}{*}{ Estrategias de aprendizaje } & \multirow{3}{*}{$50,19(0,075)$} & NA & $22,88 \%$ & \multirow{3}{*}{-} & \multirow{3}{*}{-} \\
\hline & & $\mathrm{NM}$ & $18,73 \%$ & & \\
\hline & & NB & $12,57 \%$ & & \\
\hline \multirow{3}{*}{$\begin{array}{l}\text { Desajuste en la Autoevaluación } \\
\text { Sin Ayuda }\end{array}$} & \multirow{3}{*}{$1,96(0,374)$} & NAD & $23,53 \%$ & \multirow{3}{*}{-} & \multirow{3}{*}{-} \\
\hline & & NMD & $17,69 \%$ & & \\
\hline & & NBD & $14,26 \%$ & & \\
\hline \multirow{3}{*}{$\begin{array}{c}\text { Desajuste en la Autoevaluación } \\
\text { Con Ayuda }\end{array}$} & \multirow{3}{*}{$1,36(0,507)$} & NAD & $22,05 \%$ & \multirow{3}{*}{-} & \multirow{3}{*}{-} \\
\hline & & NMD & $17,66 \%$ & & \\
\hline & & NBD & $13,99 \%$ & & \\
\hline \multicolumn{6}{|l|}{ Detección Errores Con Ayuda } \\
\hline Variable de categorización & $\chi^{2}(p)$ & Categorías & Media & Diferencia & $p$ \\
\hline \multirow{3}{*}{ Motivación } & \multirow{3}{*}{$8,37(0,015)$} & MAA & $47,12 \%$ & \multirow{3}{*}{ MAA-D } & \multirow{3}{*}{$0,00^{* 1}$} \\
\hline & & MAR & $45,60 \%$ & & \\
\hline & & $\mathrm{DM}$ & $30,55 \%$ & & \\
\hline \multirow{3}{*}{ Estrategias de aprendizaje } & \multirow{3}{*}{$8,85(0,012)$} & NA & $48,31 \%$ & \multirow{3}{*}{ NA-NB } & \multirow{3}{*}{$0,00^{* 1}$} \\
\hline & & $\mathrm{NM}$ & $40,31 \%$ & & \\
\hline & & NB & $31,62 \%$ & & \\
\hline \multirow{3}{*}{$\begin{array}{l}\text { Desajuste en la Autoevaluación } \\
\text { Sin Ayuda }\end{array}$} & \multirow{3}{*}{$2,81(0,245)$} & NAD & $43,80 \%$ & \multirow{3}{*}{-} & \multirow{3}{*}{-} \\
\hline & & NMD & $44,50 \%$ & & \\
\hline & & NBD & $34,55 \%$ & & \\
\hline \multirow{3}{*}{$\begin{array}{c}\text { Desajuste en la Autoevaluación } \\
\text { Con Ayuda }\end{array}$} & \multirow{3}{*}{$4,85(0,088)$} & NAD & $47,58 \%$ & & \\
\hline & & NMD & $40,11 \%$ & - & - \\
\hline & & NBD & $31,40 \%$ & & \\
\hline
\end{tabular}

MAA: motivación alta para el aprendizaje, MAR: motivación alta para el rendimiento, DM: desmotivación, NA: nivel alto, NM: nivel medio, NB: nivel bajo, NAD: nivel alto desajuste, NMD: nivel medio desajuste, NBD: nivel bajo desajuste. ${ }^{1}$ U-test con corrección de Bonferroni

El análisis diferencial demuestra que tanto para las variables cognitivo-motivacionales, como para las relacionadas con la autoevaluación (DASA y DACA), no se han encontrado diferencias estadísticamente significativas cuando los estudiantes detectan sus errores sin ayuda (ver Tabla 2). De acuerdo con los resultados correlacionales presentados anteriormente, en el caso de la DECA el análisis diferencial arroja un mayor núme- ro de diferencias estadísticamente significativas. Las variables cognitivo-motivacionales presentan un efecto interesante en la detección de errores que efectúan los estudiantes. En lo referente a la motivación, se dan diferencias significativas $(p=0,00)$ entre aquellos alumnos con motivación alta para el aprendizaje, que detectan el 47,12\% de sus errores y los estudiantes desmotivados que encuentran únicamente el 30,55\% de los mismos. 
Por otro lado mientras que, los alumnos con nivel alto en estrategias de aprendizaje localizan el $48,31 \%$ de los errores, aquellos estudiantes con nivel bajo detectan el $31,62 \%$, siendo esta diferencia estadísticamente significativa $(p=0,00)$. Por último, no se encuentran diferencias cuando se toman como variables categóricas el desajuste en la autoevaluación con y sin ayuda del guion de evaluación.

\section{Discusión y conclusiones}

De acuerdo con los hallazgos previos (García y Pintrich, 1994; Martín, Bueno y Ramírez, 2010; Pintrich y De Groot, 1990), los análisis correlacionales de la presente investigación muestran relación entre las variables motivacionales y las cognitivas. Aunque cabría esperar que existiese una relación entre la facilidad que tienen los estudiantes para autoevaluarse y la gestión que hacen de sus propios errores, los resultados indican que no existe correlación entre estas dos variables. Este hecho podría indicar que, cuando los estudiantes se autoevalúan, no piensan en los errores concretos que ha podido cometer sino que llevan a cabo un análisis más superficial y, tal vez, basado en factores externos al propio trabajo que están valorando. Existen resultados previos que indican que los sujetos no siempre fundamentan sus juicios en la propia tarea a evaluar, sino en su dominio de la materia en la que está basada la tarea. Este condicionamiento genera una auto-clasificación de los individuos como expertos o no expertos que influye en su valoración más que la propia tarea evaluada (Glenberg y Epstein, 1987).

El efecto de la rúbricas y guiones de evaluación en el aprendizaje ha sido estudiado anteriormente (Panadero, Alonso y Huertas, 2012; Panadero y Romero, 2014). Estas investigaciones, indican que se trata de instrumentos de gran utilidad para que los estudiantes comprendan los criterios de evaluación y puedan autoevaluarse con mayor precisión, siempre que se empleen en las condiciones adecuadas. Aunque estos autores han encontrado que la utilización de rúbricas puede aumentar el estrés de los estudiantes, sus investigaciones también demuestran que su uso mejora la precisión de los mismos al valorar sus trabajos y sus diferentes actuaciones. Además, han observado un incremento en la presencia de las estrategias de aprendizaje cuando las rúbricas se incorporan al proceso de enseñanza-aprendizaje (Panadero y Romero, 2014).

En un trabajo anterior, se comprobó que las tareas de autoevaluación y detección de errores por parte del alumnado mejoran significativamente gracias al uso de guiones de evaluación ( $\mathrm{Za}$ mora et al., 2018). Los resultados que se encontraron en la presente investigación, indican una correlación significativa entre las variables DASA y DACA. De la misma forma también existe este tipo de correlación entre DESA y DECA, lo que respalda los resultados comentados anteriormente (Zamora et al., 2018). Por tanto, el uso del guion de evaluación mejora ambas tareas, pero la habilidad de partida de los estudiantes para llevarlas a cabo también influye de manera importante en el aprovechamiento de este recurso.

La facilidad con que los estudiantes detectan sus errores presenta una influencia interesante en cuanto a los efectos del uso del guion de evaluación. Cuando los estudiantes llevan a cabo la detección de errores sin usar el guion, tan solo la regulación del esfuerzo y la autoeficacia presentan correlaciones significativas $\left(0,19^{* *}\right.$ y $\left.0,20^{* *}\right)$ con la variable DESA. Sin embargo, la utilización del guion de evaluación provoca una relación más intensa entre dificultad con la que los estudiantes detectan sus errores y ambas variables $\left(0,26^{* *}\right.$ y $\left.0,34^{* *}\right)$. Además, se produce la aparición de más variables correlacionadas que sin la utilización de la misma no estaban correlacionadas como son el valor de la tarea $\left(0,21^{* *}\right)$ y la estrategia de elaboración $\left(0,29^{* *}\right)$. Por tanto, a pesar de tratarse de tareas muy similares, parece que el hecho de utilizar el guion pone en movimiento más estrategias de aprendizaje que cuando no se utilizan estos instrumentos. Estos resultados, apuntan en la misma dirección que los obtenidos por Panadero y Romero (2014). Por ejemplo, se han encontrado diferencias significativas en los análisis diferenciales entre el grupo de estudiantes con motivación alta y baja en la detección de errores cuando los estudiantes reciben la ayuda del guion. También se han manifestado diferencias, entre los estudiantes con niveles alto y bajo en el uso de estrategias de aprendizaje.

De acuerdo con estos resultados, existe una mayor correlación entre las variables de estrategias de aprendizaje y motivación y las variables de detección de errores que las correspondientes a la autoevaluación. Este hallazgo podría apuntar a que los estudiantes de ciencias llevan a cabo la tarea de detección de errores de una forma más profunda que la de autoevaluación poniendo en juego, por tanto, un mayor número de estrategias cognitivo-motivacionales.

Por otro lado, los resultados del análisis diferencial indican que las estrategias cognitivo-motivacionales son importantes cuando los estudiantes emplean los guiones de evaluación para detectar sus propios errores. Concretamente, los sujetos con una motivación alta detectan más errores en promedio $(47,12 \%)$ que los alumnos desmotivados (30,55\%). Lo mismo sucede con las estrategias de aprendizaje. En este caso, aquellos alumnos que utilizan diversas estrategias a la hora de aprender, detecta más errores $(48,31 \%)$ que aquellos estudiantes que carecen de este tipo de estrategias $(31,62 \%)$.

Finalmente, conviene destacar que el hecho de que los estudiantes tiendan a sobreestimar su calificación se ha observado en prácticamente todos los estudios realizados hasta la fecha, tanto en otras disciplinas como en el caso particular de los estudiantes de ciencias (Hawker, Dylesk y Rickley, 2016; Lindsey y Nagel, 2015; Mathabathe y Potgieter, 2014). En este sentido, los resultados que se presentan en este artículo parecen indicar que las precisiones en los juicios de los estudiantes a la hora de autoevaluarse mejorarían si mediante rúbricas, guiones de evaluación o cualquier otro procedimiento, los estudiantes recibiesen instrucción para detectar sus errores y, por consiguiente, pudieran basar sus juicios en ellos.

En resumen, los resultados de este estudio muestran que hay una relación entre la capacidad de los estudiantes para detectar sus propios errores y las variables estratégico-motivacionales, lo que demuestra la importancia de la detección de errores en el ciclo de autorregulación del aprendizaje. Sin embargo, la relación de estas variables con los proceso de autoevaluación es mucho menos intensa. De acuerdo con los resultados, la utilización de un guion de evaluación requiere la movilización de estrategias cognitivo-motivacionales que no están presentes en las mismas tareas cuando esta no se utiliza. En la línea de los hallazgos de Panadero y Romero (2014), el uso de los guiones facilita tanto la precisión en los juicios de auto-calificación de los estudiantes como en los procesos de localización de sus errores por parte de los estudiantes. Los resultados de este trabajo indican que los aspectos cruciales a la hora de que los estudiantes detecten sus errores son, principalmente, su motivación y su nivel uso de estrategias de aprendizaje. A la luz de estos hallazgos, se propone para sucesivas investigaciones profundizar en las relaciones entre las variables del estudio desarrollando modelos de ecuaciones estructurales que ayuden a cuantificar el peso de sus distintas contribuciones. 


\section{Referencias bibliográficas}

Alonso, J., y Panadero, E. (2010). Effects of self-assessment scripts on self-regulation and learning. Infancia y aprendizaje, 33(3), 385-397. DOI: 10.1174/021037010792215145

Astolfi, J. P. (1999). El "error", un medio para enseñar. Sevilla: Diada Editora

Barca-Lozano, A., Almeida, L., Porto-Rioboo, A. M.․․, Peralbo-Uzquiano, M., y Brenlla-Blanco, J. (2012). Motivación escolar y rendimiento: impacto de metas académicas, de estrategias de aprendizaje y autoeficacia. Anales de Psicología, 28(3), 848-859. DOI:10.6018/analesps.28.3.156221

Baars, M., Vink, S., van Gog, T., de Bruin, A., y Paas, F. (2014). Effects of training self-assessment and using assessment standards on retrospective and prospective monitoring of problem solving. Learning and Instruction, 33, 92-107. DOI: 10.1016/j.learninstruc.2014.04.004

Baas, D., Castelijns, J., Vermeulen, M., Martens, R., y Segers, M. (2015). The relation between Assessment for Learning and elementary students' cognitive and metacognitive strategy use. British Journal of Educational Psychology, 85, 33-46. DOI: 10.1111/bjep.12058

Black, P., y William, D. (1998). Assessment and classroom learning. Assessment in Education, 5(1), 7-74. DOI:10.1080/0969595980050102

Briceño, M. T. (2009). El uso del error en los ambientes de aprendizaje: una visión transdisciplinaria. Revista de Teoría y Didáctica de las Ciencias Sociales, 14, 9-28.

Cueli, M., García, T., y González-Castro, P. (2013). Autorregulación y rendimiento académico en Matemáticas. Aula Abierta, 41(1), 39-48.

Field, A. (2009). Discovering statistics using SPSS. Londres: SAGE.

García, T., y Pintrich, P. R. (1994). Regulating motivation and cognition in the classroom: The role of self-schemas and self-regulatory strategies. En D. H. Schunk y B. J. Zimmerman (Eds.), Self-regulation of learning and performance: Issues and educational applications (pp. 127-154). Hillsdale, New Jersey: Lawrence Erlbaum Associates.

Glenberg, A., y Epstein, W. (1987). Inexpert calibration of comprehension. Memory and Cognition, 15, 84-93.

González, A., y Paolini, P. V. (2015). Perceived autonomy-support, Expectancy, Value, metacognitive strategies and performance in chemistry: a structural equation model in undergraduates. Chemistry Education Research and Practice, 16, 640-653. DOI: 10.1039/C5RP00058K

Hattie, J. (2013). Calibration and confidence: Where to next? Learning and Instruction, 24, 62-66. doi: 10.1016/j.learninstruc.2012.05.009

Hawker, M., Dysleski, L., y Rickey, D. (2016). Investigating general chemistry students' metacognitive monitoring of their exam performance by measuring postdiction accuracies over time. Journal of Chemical Education, 93(5), 832-840. DOI: 10.1021/acs.jchemed.5b00705

Henderson, C., y Harper, K. (2009). Quiz corrections: improving learning by encouraging students to reflect on their mistakes. The Physics Teacher, 47, 581-586. DOI: 10.1119/1.3264589

Lindsey, B., y Nagel, M. L. (2015). Do students know what they know? Exploring the accuracy of students' self-assessments. Physical Review Special Topics-Physics Education Research, 11(2), 1-11. DOI: 10.1103/PhysRevSTPER.11.020103

Martín, Ma․, Bueno, J.A., y Ramírez, Ma․ (2010). Autorregulación y rendimiento académico en Matemáticas. Aula Abierta, 38(1), 59-70.
Mathabathe, K., y Potgieger, M. (2014). Metacognitive monitoring and learning gain in foundation chemistry. Chemistry Education Research and Practice, 15, 94-104. DOI: 10.1039/C3RP00119A

Mason, A., y Singh, C. (2010). Do advanced physics students learn from their mistakes without explicit intervention? American Journal of Physics, 7(7), 760-767. DOI: 10.1119/1.3318805

Nunziati, G. (1990). Pour construite un dispositif d'évaluation formatrice. Cahiers pédagogiques, 280, 47-64.

Núñez, J. C., Solano, P., González-Pienda, J. A., y Rosário, P. (2006). El aprendizaje autorregulado como medio y meta en la educación. Papeles del Psicólogo, 27(3), 139-146.

Panadero, E., y Alonso, J. (2013). Self-assessment: theoretical and practical connotations. When it happens, how is it acquired and what to do to develop it in our students. Electronic Journal of Research in Educational Psychology, 11, 551-576. DOI: 10.14204/ejrep.30.12200

Panadero, E., Alonso, J., y Huertas, J. A. (2012). Rubrics and self-assessment scripts effects on self-regulation, learning and self-efficacy in secondary education. Learning and Individual Differences, 22, 806-813. DOI: 10.1016/j.lindif.2012.04.007

Panadero, E., y Jonsson, A. (2013). The use of scoring rubrics for formative assessment purposes revisited: A review. Educational Research Review, 9, 129-144. DOI: 10.1016/j.edurev.2013.01.002

Panadero, E., y Romero, M. (2014). To rubric or not to rubric? The effects of self-assessment on self-regulation. performance and self-efficacy. Assessment in Education: Principles, Policy y Practice, 21(2), 133-148. DOI: 10.1080/0969594X.2013.877872

Pintrich, P. R., y De Groot, E. (1990). Motivational and self-regulated learning components of classroom academic-performance. Journal of Educational Psychology, 82(1), 33-40.

Pintrich, P. R., y García, T. (1991). Student goal orientation and self-regulation in the college classroom. En M. Maehr y P.R. Pintrich (Eds.), Advances in motivation and achievement: Goals and self-regulatory processes (pp. 371-402). Greenwich. CT: TAI.

Pintrich, P. R., Smith, D., García, T., y McKeachie, W. (1991). A manual for the use of the Motivated Strategies for Learning Questionnaire (MSLQ). National Center for Research to Improve Postsecondary Teaching and Learning: University of Michigan.

Rosário, P. (2004). Estudar o Estudar: As (Des)venturas do Testas. Porto: Porto Editora.

Schank, R. (1997). Virtual learning: a revolutionary approach to building a highly skilled workforce. New York: McGraw-Hill.

Schunk, D., y Zimmerman, B. (2013). Self-regulation and Learning. En W. M. Reynolds y G. E. Miller (Eds.), Handbook of psycholo$g y$ (pp. 45-68). NJ: Wiley.

Yerushalmi, E., Cohen, E., Mason, A., y Singh, C. (2012a). What do students do when asked to diagnose their mistakes? Does it help them? II. A more typical quiz context. Physics Education Research, 8(2), 1-12. DOI: 10.1103/PhysRevSTPER.8.020110

Yerushalmi, E., Cohen, E., Mason, A., y Singh, C. (2012b). What do students do when asked to diagnose their mistakes? Does it help them? I. An atypical quiz context. Physics Education Research, 8(2), 1-19. DOI: 10.1103/PhysRevSTPER.8.020109

Zamora, Á., y Ardura, D. (2014). ¿En qué medida utilizan los estudiantes de Física de Bachillerato sus propios errores para aprender? Una experiencia de autorregulación en el aula de secundaria. Enseñanza de las Ciencias, 32(2), 253-268. DOI: 10.5565/rev/ensciencias.1067

Zamora, Á., Suárez, J.M. y Ardura, D. (2017). A model of the role of error detection and self- regulation in academic performance. The Journal of Educational Research, DOI: $10.1080 / 00220671.2017 .1349072$ 
Zamora, Á., Suárez, J.M., y Ardura, D. (2018). Error detection and self-assessment as mechanisms to promote self-regulation of learning among secondary education students. The Journal of Educational Research, 111(2), 175-185. DOI: $10.1080 / 00220671.2016 .1225657$
Zimmerman, B. (2000). Attaining self-regulation: a social cognitive perspective. En M. Boekaerts. P. R. Printich and M. Zeidner, Handbook of self-regulation (pp. 13-39). San Diego. CA: Academic Press. 